\title{
La construcción de la «figura» ministerial en el Perú. Una mirada multidimensional y relacional al estudio de las élites estatales
}

\section{Arthur Morenas}

Recibido: 23-ago-20

Aprobado: 18-nov-20

doi: $10.46476 /$ ra.v1i 2.55

\section{Resumen}

El Estado y sus élites han sido objeto de varios estudios, mediante los cuales se renueva y profundiza el conocimiento sobre las formas de gobierno y de acceso a los puestos de la alta administración pública del Perú. Este análisis se centra en los ministros nombrados en las principales carteras, encargadas de los temas de desarrollo económico y social entre 2001 y 2020. En ese sentido, se propone profundizar en el conocimiento de una categoría específica de agente estatal, fuertemente construida a nivel social y simbólico. El trabajo prosopográfico y el Análisis de Correspondencias Múltiples, con el enfoque multidimensional y relacional subyacente, permiten objetivar un poco más el peso de las trayectorias y perfiles profesionales de los ministros peruanos en el acceso a estos cargos político-administrativos. El artículo también sugiere la hipótesis de un espacio fuertemente organizado en torno a dimensiones sociales y generacionales, que no son ajenas a las dinámicas de construcción socioprofesional que tienen lugar en otros espacios. Por último, el trabajo permite situar en el espacio de los ministros peruanos los diferentes ministerios estudiados, así como las presidencias (Toledo, García, Humala, Kuczynski y Vizcarra) para identificar las características propias de estos ministerios y presidencias.

Palabras clave: ministros, élites políticas, élites estatales, tecnocracia, Estado, poder. 


\section{Abstract}

The State and its elites were the subject of several studies that have updated and improved our knowledge of forms of governments and access to senior positions in Peru's public sector. By focusing on the ministers appointed in Peru between 2001 and 2020 to the main portfolios responsible for economic and social development, this paper provides a more in-depth understanding of a specific type of state agent with deep roots in the social and symbolic spheres. Through prosopography and multiple correspondence analysis, based on an underlying multidimensional, relational approach, it becomes possible to more objectively determine the degree of importance of Peruvian ministers' professional trajectories and profiles in gaining access to these political-administrative positions. The paper also proposes the hypothesis of a space that is heavily organized around social and generational dimensions, which are often tied to the dynamics of socio-professional construction that occur in other spaces. Finally, the work helps situate the different ministerial and presidential administrations (Toledo, Garcia, Humala, Kuczynski, Vizcarra) studied within the Peruvian ministers' socio-professional space, in order to identify the salient characteristics specific to these ministries and presidencies.

Keywords: ministers, political elites, state elites, technocracy, state, power.

\section{Resumo}

O Estado e as suas elites têm sido objeto de vários estudos, renovando e aprofundando o conhecimento das formas de governo e do acesso a cargos na administração pública sênior do Peru. Centrando-se nos ministros, entre 2001 e 2020 no Peru, nomeados nas principais pastas responsáveis pelas questões de desenvolvimento econômico e social, o artigo propõe aprofundar o conhecimento de uma categoria específica, fortemente construída a nível social e simbólico, de agente estatal. O trabalho prosopográfico e a Análise de Correspondência Múltipla, e a abordagem multidimensional e relacional subjacente, permite objetivar um pouco mais o peso das trajetórias e perfis profissionais dos ministros peruanos no acesso a estas posições político-administrativas. $O$ trabalho sugere também a hipótese de um espaço fortemente organizado em torno de dimensões sociais e geracionais, que não são alheias às dinâmicas de construção sócio profissional que têm lugar noutros espaços. Finalmente, o trabalho permite situar, no espaço sócio profissional dos ministros peruanos, os diferentes ministérios estudados, bem como as presidências (Toledo, Garcia, Humala, Kuczynski, Vizcarra) a fim de identificar as características salientes específicas destes ministérios e presidências.

Palavras-chaves: Ministros, elites políticas, elites estatais, tecnocracia, Estado, poder. 


\section{Introducción}

Los nombramientos y los cambios de mando de los ministerios generan, frecuentemente, comentarios y debates. Recientemente, el nombramiento de Martín Ruggiero Garzón como ministro de Trabajo ha sido fuertemente criticado, no tanto por su edad (32 años) sino por su falta de experiencia en el sector público. $\mathrm{Si}$ bien esta falta de experiencia no es formalmente un impedimento para formar parte de un gobierno, los debates generados recuerdan que el puesto de ministro es más que un proceso administrativo, es un acto fundamentalmente político. Esta es una razón por la cual el estudio de los ministros abre perspectivas para entender no solamente el Estado sino también la institucionalización de ciertas reglas y prácticas (formales o no) que organizan la relación entre las esferas que participan, o buscan participar, en la organización política de una sociedad. En la línea con diversas propuestas académicas (Dargent, 2012a; Tanaka, 2010), el objetivo del artículo es contribuir al conocimiento del Estado peruano, desde quienes «hacen» al Estado.

La presentación de las figuras ministeriales en el Perú es frecuentemente utilizada para recordar el carácter cada vez más tecnocrático de las formas de administración pública de hoy en día (mencionando solo algunos trabajos: Conaghan, Malloy \& Abugattas, 1990; Dargent, 2012b; Grompone Velásquez, 2017; Vergara \& Encinas, 2016) pero también de épocas anteriores (ver por ejemplo: Grompone, 2016; Sala i Vila, 2016; Wise, 2003). Estos trabajos se distancian, de una u otra manera, de los análisis en términos de «captura del Estado» (Crabtree \& Durand, 2017), los cuales insisten en la circulación frecuente de los ministros y altos funcionaros entre las altas esferas del Estado y posiciones de gerencia en grandes empresas privadas (la «puerta giratoria»). Sin pretender resolver esta dualidad, podemos mencionar que los fenómenos de autonomización de grupos de tecnócratas y de influencias empresariales en los nombramientos no se oponen necesariamente (Vergara, 2012); en ese sentido, por ejemplo, existen dinámicas sectoriales y juegos de influencias cruzadas. Introduciendo una dimensión comparativa, se puede recordar que el peso creciente de los «tecnócratas» en las esferas del poder obedece a reglas y dinámicas distintas, según los países. Las estructuras y configuraciones del poder, así como coyunturas políticas, sociales o económicas, influyen fuertemente en las formas con las cuales se institucionalizan algunas figuras específicas de la «tecnocracia». Fourcade y Babb (2002) así como Dezalay y Garth (2002) muestran que los rasgos sociohistóricos de los países (la formación del Estado y las relaciones de sus agentes con otras esferas del poder, la constitución y estructuración de los espacios de producción de los saberes, técnicos y estatales, etc.) influyen fuertemente en las estrategias de construcción y legitimación de nuevas formas de gobierno. Autores como Ihl (2012) o Heredia (2015) demuestran, por su lado, la necesidad de prestar especial atención a la conjunción entre dinámicas propias de estas esferas del poder 
y elementos contextuales, para salir de la ilusión heroica que suele acompañar a la presentación de la «toma del poder» por los «tecnócratas». En otras palabras, no se puede reducir la presencia en el poder de ciertos grupos de individuos a la necesidad de resolver un "problema», cumplir «buenas prácticas» o responder a intereses privados. La «figura» ministerial es un objeto en constante disputa y está sujeta a múltiples cambios, los cuales son claros en el caso peruano: desde el creciente peso de la economía y los estudios en los Estados Unidos (Dargent, 2012b; Vela, Becerra, García, \& Roca, 2014; Villagarcia, 2014) hasta la importancia de formas de socialización y conformación de un habitus en la universidad o los organismos internacionales (Vergara, 2012; Vergara \& Encinas, 2016).

De manera general, los trabajos mencionados sobre el perfil de las élites estatales en el Perú ponen énfasis en la fuerte disminución del perfil «político» (entendido como política partidaria y cargos electorales) de los ministros, lo cual suele ser analizado como la correlación lógica del peso creciente de los tecnócratas en los espacios de producción de las políticas públicas en el país. La cuestión de la oposición entre «tecnócrata»y "político» es compleja, ya que hasta los actos administrativos aparentemente más técnicos incorporan factores y prácticas de corte político (Benites Alvarado \& Hurtado Paz, 2018). Si el factor político es importante hasta en los niveles más administrativos del Estado peruano, es poco probable que el manejo de los asuntos públicos en el ejecutivo sea solamente cuestión de gestiones técnicas. Otros ejemplos nacionales muestran que la dimensión política (en su acepción partidaria) no es necesariamente opuesta a la valorización de los perfiles «tecnocráticos». En un trabajo sobre la composición de los gabinetes de la Concertación en Chile, entre 1990 y 2010, Bustamante (2013) demuestra que tener un alto perfil académico o tecnocrático no basta para acceder, y tampoco durar, en los puestos gubernamentales más importantes: pertenecer y trabajar en relación estrecha con un partido de la Concertación es una condición importante para lograr tal incorporación al ejecutivo chileno. Por su lado, Dulong recuerda que los agentes que participaron en la construcción de la figura del tecnócrata en Francia lo hicieron, en parte, mediante la competencia política, tratando de «transformar posiciones de poder político inscribiendo la competencia económica en la definición de algunos roles políticos» (traducción personal de Dulong, 1996, p. 126). La participación política de Pedro Pablo Kuczynski desde 2011 ilustra en el caso peruano esta relación, más estrecha que lo usualmente presentado, entre campo político y campo tecnocrático. La ausencia de vínculos claros entre participación política partidaria o electiva e institucionalización de la figura del tecnócrata puede, en este sentido, ser entendida como una consecuencia de la debilidad partidaria en el Perú (Tanaka, Vera Rojas, \& Barrenechea, 2009) así como de la difícil conversión del capital tecnocrático en capital político. 
Interesarse en el perfil de los ministros en el Perú permite aproximarse al conocimiento de los capitales y recursos necesarios para acceder a estos altos puestos de la administración pública, para entender al mismo tiempo, las dinámicas que acompañan la estructuración de las élites del Estado. Las hipótesis que organizan este trabajo se articulan alrededor de una serie de tres interrogantes. Primero, se trata de entender cuáles son las dinámicas que acompañan la construcción del perfil ministerial en el Perú. Nuestra principal hipótesis al respecto es que la construcción de la figura ministerial no se explica solamente por la valorización de un capital cultural «técnico» (alto nivel de estudios, por ejemplo) y un alejamiento de la militancia y de los cargos político-electivos. Estas características son, sin lugar a duda, importantes para entender las diferencias que pueden existir en los perfiles de los ministros, pero es necesario tomar en cuenta el conjunto de capitales adquiridos mediante trayectorias socio-profesionales, realizadas en diferentes espacios. Una segunda hipótesis al respecto es que existe una cierta relación de homología entre las dinámicas que se dan en la composición de estos altos cargos del Estado y dinámicas en las estrategias de formación y legitimación de las élites económicas. Esta relación de homología entre campo estatal y el campo económico dan cuenta, a su vez, de un espacio socialmente estructurado. En ese sentido, la composición de capital social, cultural y económico de grupos importantes de ministros es socialmente elitista. Luego, nos preguntaremos si los diferentes perfiles de ministros suelen asociarse a algunas carteras especificas o prevalecer durante alguna presidencia. Nuestra hipótesis al respecto es que la composición de capital asociada a cada ministerio depende en gran parte de las trayectorias de los ministros, entre sector público y privado. De la misma manera, el contexto político y la posición de los presidentes en la estructura de relaciones que constituyen el poder en el Perú contribuyen a orientar el perfil de los ministros.

En un primer momento, haremos una breve presentación del marco teórico y la metodología. Luego, mostraremos que las formas de accesos a puestos ministeriales en el Perú están fuertemente asociadas no solamente a perfiles «técnicos» sino también a un conjunto de características, saberes y prácticas, proveniente de los espacios de la competencia económica. Para finalizar, trataremos de identificar las principales características asociadas a los ministerios estudiados y ver si las diferentes presidencias, en el transcurso de los años 2000, se caracterizaron por abarcar perfiles ministeriales similares o si existen, al contrario, diferencias en la selección de los ministros, según los presidentes que gobernaron entre 2001 e inicios del $2020{ }^{1}$

1. El estudio no incluye a los ministros nombrados en el efímero gabinete Cateriano, ni tampoco en el gabinete Martos. 


\section{Estudiar a los ministros desde una perspectiva multidimensional y relacional}

Pensar un espacio social de manera multidimensional es recordar el carácter múltiple y complejo de las relaciones entre individuos que participan en este. Estudiar las lógicas constitutivas de los grupos ministeriales permite aproximarse a la manera con la cual se construyen los espacios del poder y algunas de sus principales figuras: el «buen gestor», el «político», el «tecnócrata», pero también el «intelectual» o el «emprendedor exitoso», etc. La dimensión relacional implica que las características de un grupo deben ser entendidas en su relación con las características de otros grupos. La cantidad y composición de capital (económico, cultural o social) de cada individuo que participa en el espacio permite objetivar una estructura de posiciones y por lo tanto una estructura de relaciones entre estos individuos.

Esta perspectiva suele ser asociada al estudio de las clases sociales, de las prácticas y preferencias culturales, desde los trabajos fundadores de Bourdieu y Saint Martin (Bourdieu \& Saint Martin, 1976; Bourdieu, 2017) hasta trabajos más actuales sobre Chile, Gran Bretaña o Perú (Gayo, Teitelboim, \& Méndez, 2013; Savage y al., 2013; Zárate \& Rentería, 2019). Desde esta perspectiva, existe toda una tradición de estudio sobre las élites políticas, económicas o intelectuales, así como avances importantes dentro del estudio del Estado y de las políticas públicas (Dubois, 2015; Poupeau, O’Neill, Cortinas Muñoz, Coeurdray, \& Benites-Gambirazio, 2020). Para limitarnos aquí a la temática de las élites políticas y de los ministros, el análisis multidimensional permite objetivar las diferentes formas de capitales asociados a importantes posiciones de poder. En países como Francia, donde la alta burocracia está fuertemente institucionalizada, estos análisis permiten distinguir entre «entrada directa al centro» $\mathrm{y}$ «entrada indirecta» (Behr \& Michon, 2013). La primera corresponde a ministros provenientes de la alta burocracia, quienes suelen pertenecer a uno de los principales partidos y alcanzan posiciones ministeriales después de trabajar en los despachos ministeriales, mientras que la segunda describe una amplia trayectoria electoral, con la acumulación de mandatos (locales y nacionales), previos al nombramiento en un ministerio. Evidentemente, en un país como el Perú donde la alta burocracia resulta poco institucionalizada y los partidos políticos no constituyen vehículos estables de carrera política (Zavaleta, 2014), adoptar una perspectiva multidimensional implica construir una serie de variables que no se limitan al nivel educativo, a las experiencias en el sector público o a la pertenencia de un partido político. Las trayectorias profesionales, el tipo de carrera en el sector público o privado, la cercanía con grupos de interés, actividades académicas o grandes consultorías son dimensiones importantes en la constitución de las figuras ministeriales (un ejemplo interesante, sobre una política pública especifica: Oré \& Rap, 2009). El trabajo se centra en los ministerios cuyas principales misiones tienen que ver con el desarrollo económico, 
productivo y social. ${ }^{2}$ Se trata de buscar cierta homogeneidad, una condición para que el estudio de un espacio social tenga sentido. ${ }^{3}$ Se constituyó, mediante una prosopografía, una base de datos compuesta de 90 personas que ocuparon, entre el 2001 y 2020, una de las siguientes carteras: Ministerio de Economía y Finanzas (MEF), Ministerio de Comercio y Turismo (Mincetur), Ministerio de Desarrollo e Inclusión Social (Midis), Ministerio de Agricultura (Minagri), Ministerio de Producción (Produce), Ministerio de Energía y Minas (Minem) y Ministerio de Vivienda (Vivienda). A partir de la base de datos, se construyeron 22 variables, con un total de 71 modalidades. Para que la constitución de los ejes no esté influenciada por modalidades «raras», es decir compartidas por una cantidad muy limitada de individuos, 6 modalidades han sido puestas como «ilustrativas»; por lo tanto, el total de modalidades activas es de $65 .{ }^{4}$

La principal herramienta de trabajo ha sido el Análisis de Correspondencias Múltiples (ACM), una técnica estadística que permite resumir la información de un grupo en ejes factoriales, y así objetivar las cercanías o divergencias entre las variables y los individuos. El Análisis de Correspondencias Múltiples tiene la ventaja de permitir trabajar sobre un gran número de variables (cualitativas), y es por lo tanto una herramienta bastante útil para un razonamiento multidimensional y relacional. La proyección geométrica de los resultados permite, asimismo, una visualización intuitiva de los principales resultados, lo que presentaremos a continuación.

\section{Estructura socio-profesional de las posiciones ministeriales en el Perú}

El Análisis de Correspondencias Múltiples se realizó a través del programa SPAD, el cual permite, además, representar gráficamente los principales resultados (proyección geométrica de las variables). La proyección geométrica consiste en resumir, en un plano factorial (dos ejes), las relaciones estadísticas entre variables

2. No se incluyó al Ministerio de Trabajo, en gran parte porque el perfil general de los ministros del trabajo suele ser asociado a abogados (Villagarcia, 2014). Los abogados y las profesiones jurídicas tienen una importancia fundamental en la institucionalización del Estado en el Perú. No obstante, los espacios profesionales de las carreras jurídicas tienen propiedades fuertemente marcadas (carreras universitarias, trayectorias profesionales e instituciones de legitimación), las cuales podían crear un sesgo en el análisis.

3. Incluir, por ejemplo, otros ministerios (salud, relaciones exteriores u otros) hubiera introducido un sesgo, en gran parte sectorial, en el análisis.

4. El Análisis de Correspondencias Múltiples especifico (COREM) permite diferenciar, dentro de las mismas variables activas, entre modalidades activas e ilustrativas. Las modalidades ilustrativas no participan en la construcción de los ejes, lo que permite integrarlas en el análisis sin alterar la constitución de los ejes. Además, hemos incluido en el análisis, de manera ilustrativa, variables socio-generacionales, pero también el cargo ministerial que ocuparon los 90 individuos, así que la(s) presidencia(s) durante la cual estuvieron en el gabinete. Ver Anexo Metodológico para más detalles: https:// seafile.unistra.fr/f/ca0895c67fe045159544/ 
activas. El ACM determina ejes sintéticos, los cuales expresan las principales diferencias y cercanías entre las propiedades y características de los individuos estudiados. La proyección geométrica permite una representación gráfica de estos ejes. El primer y segundo eje ( 1 y 2 ) son los que concentran las principales variaciones (expresadas en porcentajes de inercia) entre variables activas, pero se pueden proyectar otros ejes (por ejemplo, un plano factorial con los ejes 3 y 4 ). Este trabajo se limita aquí a presentar los dos primeros ejes ( 1 y 2), los cuales tienen inercias significativas y ofrecen una lectura interesante de las dinámicas de diferenciación del perfil de los ministros. La proyección de las variables activas (Gráfico 1) permite una interpretación de las principales diferencias y cercanías que existen en el conjunto de modalidades activas. Las modalidades que suelen ser compartidas por un grupo de individuos aparecen cercanas en el gráfico y, por el contrario, la distancia entre modalidades proyectadas expresa una diferenciación entre estas. Los dos ejes (horizontal y vertical) de la proyección sintetizan dos principios de diferenciación entre variables activas. Las variables se distribuyen en cada uno de los ejes en función al peso que tienen en la constitución del eje (relación estadística determinada por el ACM). De esta manera, las variables que participan mucho en la construcción del eje son proyectadas en una u otra extremidad, mientras que las variables que tienen poco peso aparecen más cercanas al origen (0) del eje (para una presentación más detallada del ACM y de sus fundamentos matemáticos: Le Roux, 2014; Roux \& Rouanet, 2009).

El eje 1 (horizontal, $11.2 \%$ de la inercia total) establece claramente una distinción entre dos perfiles socio-profesionales que han tenido y tienen cierta importancia en la constitución del Estado en el Perú: los ingenieros y los economistas. Este eje horizontal $\left(\mathrm{n}^{\circ} 1\right)$ da cuenta de una lógica histórico-sectorial propia de algunos ministerios (los economistas en el MEF y los ingenieros en el Minem o Minagri), pero recuerda también la oposición ya mencionada entre «tecnócrata» $\mathrm{y}$ "político». Las posiciones político-partidarias se encuentran en la parte derecha de la proyección, los estudios en el extranjero y un alto nivel educativo (doctorado) en la parte izquierda. Las modalidades que contribuyen a construir este primer eje (resaltadas en el Grafico 2) permiten profundizar esta diferenciación, la cual recoge dimensiones socio-generacionales y formas diferentes de participación en los espacios de poder en el país. De hecho, el eje horizontal $\left(n^{\circ} 1\right)$ diferencia entre perfiles fuertemente internacionalizados (en la parte izquierda) y trayectorias marcadas por conjuntos de capitales nacionales (parte derecha). 
Gráfico 1: Proyección de las variables activas - Ejes 1 \& 2 del ACM.

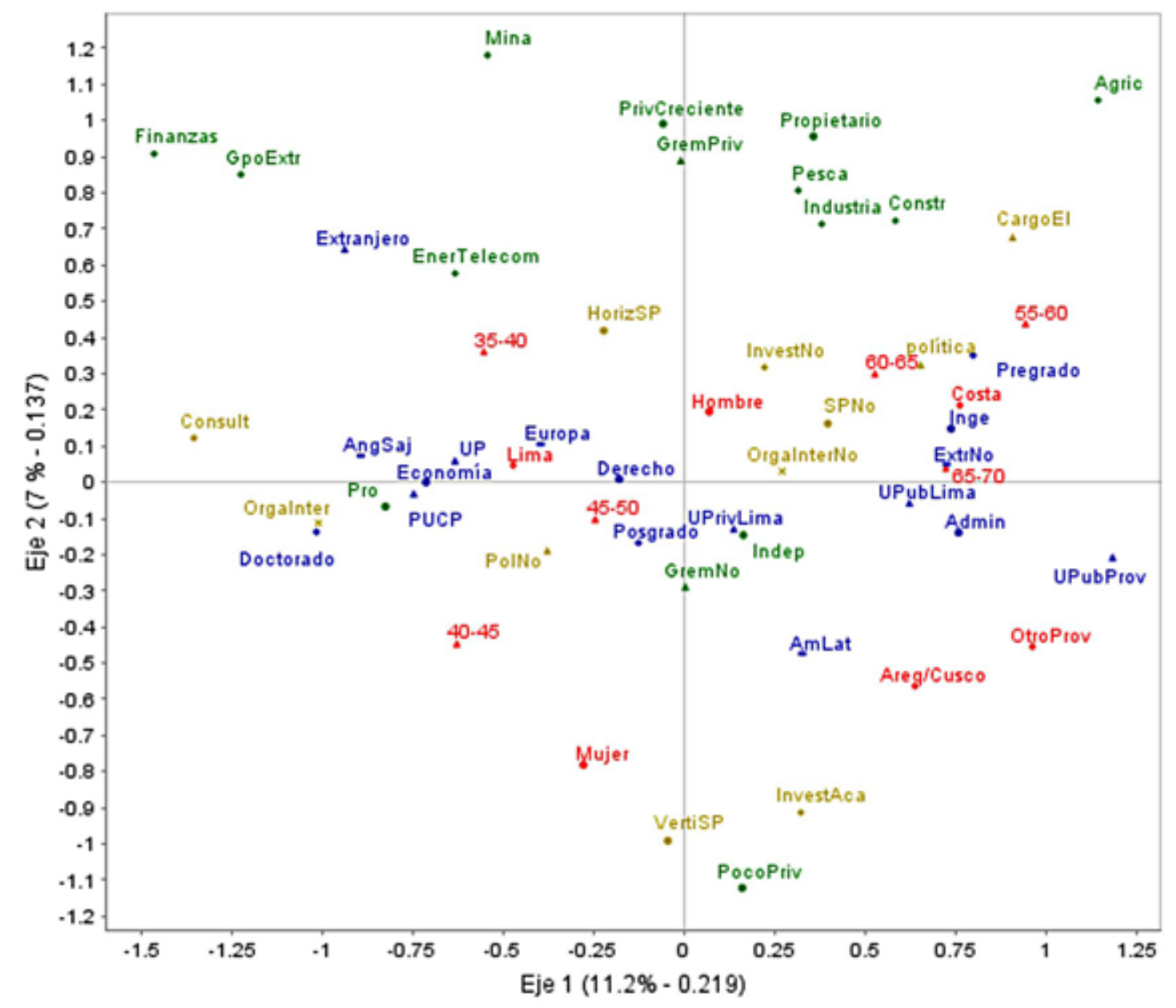

Sexo $\Delta$ EdadPrimerPuesto $\bullet$ LugarNaci $\bullet$ Carrera $\boldsymbol{\Delta}$ Universidad $\bullet$ GradoEduc

- EstudiosExtranjero - CarreraPrivada $\Delta$ Gremio $\diamond$ ActivPrivada $\odot$ TraySP $\Delta$ Política $\diamond$ InvestAca

X Orgalnter

Elaboración del gráfico por el programa SPAD 9. Recolección de los datos en base a la investigación prosopográfica.

Estas posiciones en el campo del poder peruano se acompañan de perfiles y trayectorias diferentes. Por un lado (izquierda), ministros relativamente jóvenes (40-45 años al momento de ocupar su primer puesto ministerial) nacidos en Lima, y por otro lado (derecha), individuos nacidos en provincia, que alcanzan una posición ministerial a una edad más avanzada (55-60 años). Esta primera diferenciación es multifacética. Así, la parte derecha de la proyección abarca en gran medida una dimensión histórica, asociada al papel de los ingenieros-empresarios con conexiones políticas y familiares tradicionales (Eduardo Iriarte, Álvaro Quijandría, o Rafael Quevedo, por ejemplo), pero también algunos ministros, cuya presencia en estos puestos no se debe a una multitud de conexiones políticoempresariales y familiares sino a un capital cultural técnico-nacional (ingenieros pero también administradores o abogados) valorizado generalmente a nivel local 
en organizaciones políticas o puestos en instituciones públicas (Edmer Trujillo, Glodomiro Sánchez Mejía o Susana Vilca, cada uno con sus particularidades).

De la misma manera, podemos encontrar en la parte izquierda perfiles que van desde economistas o gerentes multi-situados con fuerte presencia en instituciones financieras privadas (Pedro Pablo Kuczynski, Alfredo Thorne o Ismael Benavides) hasta figuras académicas o asociadas a carreras en organismos públicos o internacionales (Carolina Trivelli, Milton Von Hesse, María Antonieta Alva, por ejemplo). Estas múltiples dimensiones que contribuyen a organizar el primer eje recuerdan el carácter múltiple del capital político y técnico, y la necesidad de considerarlo en relación con otros tipos de capitales. De hecho, si el eje 1 recuerda la fuerte dimensión socio-generacional que existe detrás de las principales figuras ministeriales, el eje 2 (vertical, $7 \%$ de la inercia) establece una diferenciación en función a las trayectorias profesionales de los ministros y, específicamente, de las cercanías diferenciadas con los polos dominantes de los espacios de la competencia económica.

Estas diferencias se dan entre la parte superior de la proyección, la cual está asociada a posiciones importantes en el sector privado (trayectorias ascendentes, hasta ocupar cargos gerenciales, propietarios, participación en un gremio empresarial etc.) y la parte inferior, asociada a una actividad privada limitada, inexistente o ejercida de manera independiente. En cambio, es en la parte inferior de la proyección que encontramos las trayectorias «ascendentes» dentro del sector público, mientras que la parte superior se caracteriza mayormente por nombramientos directos a puestos de director o gerente (directorio de una entidad pública, gerente de un organismo público, director general o asesor ministerial, etc.). Es necesario mencionar aquí que la existencia de trayectorias «ascendentes» en el sector público no quiere decir que dichos ministros representen el arquetipo del funcionario público, ya que varios de ellos han tenido experiencias en otros rubros. Sin embargo, es posible decir que este segundo eje (vertical) retoma, con especificidades propias a la estructura institucional y profesional peruana, la diferencia mencionada entre "entrada directa al centro» y «entrada indirecta», mientras que los ministros con importantes trayectorias privadas suelen entrar directamente en el Estado mediante importantes cargos, los ministros menos cercanos a estos espacios, tienden a acumular experiencias públicas o académicas antes de acceder a las altas esferas del Estado peruano.

A continuación, presentamos los gráficos 2 y 3 en los cuales hemos resaltado las principales modalidades que entran en la construcción del primer (Grafico 2) y del segundo eje (Grafico 3). Las flechas expresan la distancia entre modalidades que participan de la construcción de una misma variable. Por ejemplo, la variable «política» (militancia política) es dicotómica y por lo tanto dos modalidades han sido proyectadas: «política» $\mathrm{y}$ «PolNo» (sin militancia política). El color de 
las modalidades proyectadas sirve para distinguir entre modalidades sociales (rojo), educativas (azul), actividades privadas (verde) y otras actividades políticas o profesionales (amarillo). La forma de las flechas no tiene significado, sirven solamente para dar más claridad a la proyección.

Gráfico 2: Trayectorias y principales variables que participan en la constitución del primer eje del ACM. (Horizontal).

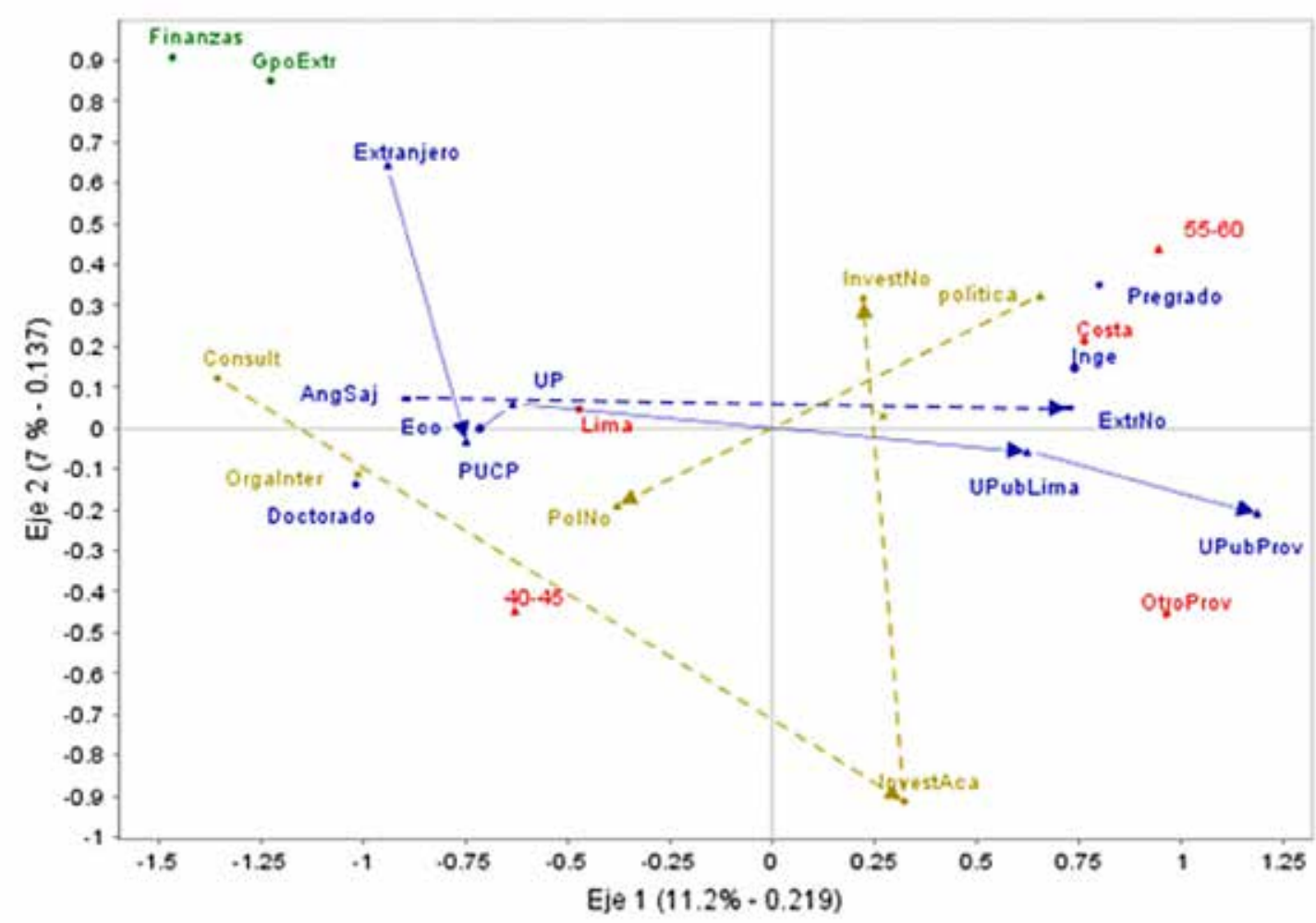

$\boldsymbol{\Delta}$ EdadPrimerPuesto $\bullet$ LugarNaci - Carrera $\mathbf{\Delta}$ Universidad $\diamond$ GradoEduc $\mathbf{m}$ EstudiosExtranjero

ActivPrivada $\Delta$ Politica $\diamond$ InvestAca $x$ Orgainter

Esta distinción entre parte superior e inferior de la proyección introduce una diferenciación entre agentes dominantes en varios espacios de poder y profesionales, cuya posición ministerial se debe en gran parte a un capital cultural (académico o técnico-burocrático) específico. Esta perspectiva permite objetivar la existencia de una polarización del perfil de los ministros en el Perú en función no solamente a su perfil académico o a sus vínculos partidarios, sino también a una composición variable de capitales (cultural, simbólico y social) que dan cuenta de posiciones diferentes en los espacios que componen el campo del poder en el Perú. Para profundizar un poco más nuestra hipótesis de un espacio socialmente estructurado, y de la reproducción, en la constitución de algunas figuras ministeriales, de dinámicas que se dieron en el campo económico peruano, hemos proyectado una serie de variables ilustrativas (que no entran en la construcción de los ejes) en una nube de puntos que representan a los diferentes ministros estudiados. El plano factorial sigue constituido por los ejes 1 (horizontal) y 2 (vertical). 
Gráfico 3: Trayectorias y principales variables que participan en la constitución del secundo eje del ACM. (Vertical).

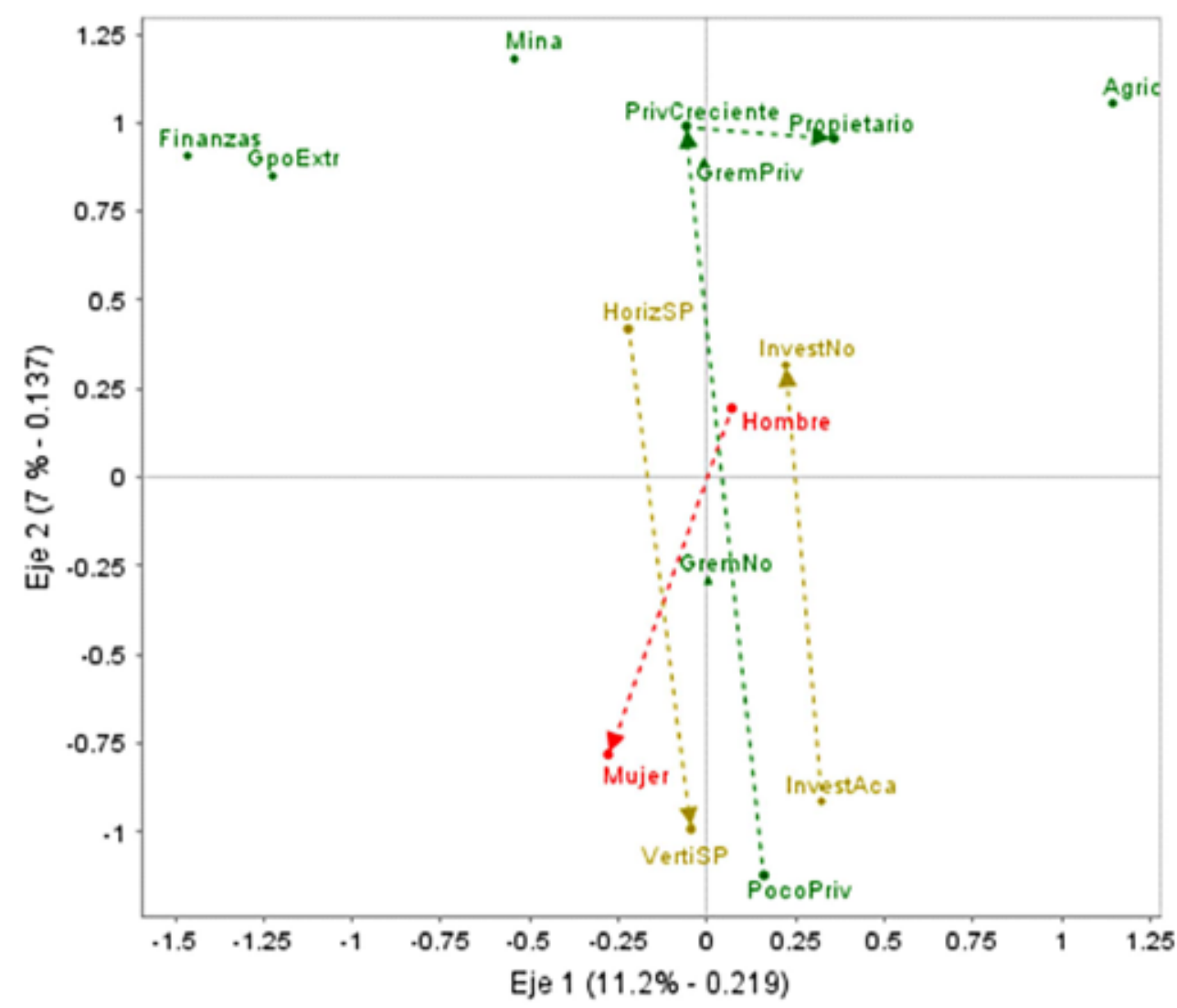

Sexo $\bullet$ CarreraPrivada $\boldsymbol{\Delta}$ Gremio $\diamond$ ActivPrivada $\odot$ TraySP $\diamond$ InvestAca

\section{Un espacio socialmente estructurado}

El hecho de que la modalidad «mujer» tenga un peso importante en la construcción del segundo eje (vertical), revela la dimensión socialmente estructurada de este, mientras que las posiciones prestigiosas y dominantes en el sector privado suelen ser asociadas a hombres, las mujeres tienden a desarrollar carreras más cercanas a esquemas técnico-intelectuales para poder llegar a posiciones ministeriales. Esto no quiere decir que ninguna mujer ministra en el Perú tenga un perfil distinto, como se pudo ver con personas como Cayetana Aljovín o Lieneke Schol. No obstante, la figura del gerente privado multi-situado, entre posiciones de poder político-administrativo y posiciones de gerencia privada permite avanzar la hipótesis de que esta figura suele ser asociada a una posición social que va más allá de un simple perfil profesional, revelando en varios aspectos la transformación de un capital familiar (tanto económico como cultural y social) en importantes posiciones de gerencia. Esta dimensión es central y está fuertemente asociada a las dinámicas de transformación de las élites económicas en el Perú, con el peso creciente de la figura del «manager» y del consultor (Durand, 2008), la cual es 
particularmente importante tratándose de los grupos económicos «establecidos» (Durand, 2017). Precisamente, recordamos que las modalidades de «trayectoria ascendente» y posiciones en un grupo de consultoría económica se ubican en el cuadro superior-izquierdo de la proyección, lo cual asocia estas figuras a las franjas de ministros más jóvenes al momento de acceder a estos puestos, fuertemente institucionalizados y, como lo veremos, socialmente elitistas.

Una variable ilustrativa indica el tipo de colegio donde los ministros estudiaron y otra corresponde a la generación (década) a la cual pertenecen. ${ }^{5}$ Para poder sintetizar la información, hemos agrupado los colegios en las siguientes modalidades: por un lado colegios elitistas de Lima, bilingües (entre estos el colegio Markham, pero también el colegio Von Humboldt) y religiosos (por ejemplo el colegio Inmaculada o Santa María Marianistas), por otro lado colegios de provincia y «otros» colegios religiosos. ${ }^{6}$ Se podría mejorar esta agrupación, matizando por ejemplo estas principales categorías en función a la información que se puede tener de estos colegios. Tratándose de la categoría "colegios en provincia», no es lo mismo, para entender y objetivar las estrategias de reproducción social y la composición de capital de los padres de los ministros, haber estudiado en el colegio San Miguel de Piura, como Javier Silva Ruete; en el colegio La Salle de Arequipa, como Carlos Leyton; que en un colegio con un peso simbólico o sociocultural diferente. Sin embargo, la categorización que hemos realizado permite introducir una primera diferenciación según el colegio, lo cual viene fortaleciendo algunas observaciones que podemos hacer en base al conjunto de variables activas. Asimismo, en cuanto a los colegios «elitistas», nos aproximamos a los criterios de diferenciación establecidos por Rentería, Grompone y Reátegui (2020), quienes recuerdan la importancia de tomar en cuenta, para el estudio de los colegio «elitistas», no solo la dimensión económica (costo de matrícula) sino también el peso históricosimbólico de estos (central en cuanto a los colegios religiosos tradicionales).

A pesar de las limitaciones ya mencionadas, la proyección de las variables ilustrativas viene a reforzar nuestra hipótesis sobre la importancia de un capital económico, cultural y social heredado en la construcción de la figura del ministro-gerente,

5. En lo ideal la variable sobre el colegio hubiera podido entrar como activa, pero debido a la dificultad de poder encontrar este tipo de información ha sido necesario considerarla como ilustrativa, limitando así la interpretación de esta variable a meras hipótesis. Ha sido posible recolectar información para dos tercios de los ministros estudiados, lo cual permite una base de trabajo interesante.

6. Los análisis de Correspondencias Múltiples son bastante sensibles a las modalidades «raras», poco frecuentes. Razón por la cual es necesario sintetizar la información. Cuando nos referimos aquí a colegios «elitistas», no estamos juzgando la calidad educativa de estos, sino que nos referimos a los colegios que suelen estar asociados a las capas más elitistas del país. Los colegios mencionados entre paréntesis son los que se encuentran con más frecuencia dentro de la base de datos: 11 veces para el colegio Inmaculada, 5 veces para el Marianista. Los colegios bilingües aparecen con menos frecuencia: 3 veces para el Markham, 2 el Von Humbolt. 
dado que las dos modalidades asociadas a los colegios más elitistas de Lima están ubicadas claramente en la parte noroeste de la proyección. Teniendo en cuenta la importancia del colegio en las estrategias familiares de reproducción social, en todos los niveles de la sociedad peruana (Román \& Ramirez Villarán, 2018), esta dimensión viene además acompañada de modalidades asociadas a figuras elitistas. Los estudios en el extranjero guardan así, a pesar de la multiplicación de las becas, una importante connotación social. Incluso programas institucionalizados como el Erasmus en la Unión Europea tienden a reproducir formas de desigualdades (Ballatore \& Blöss, 2008). Además de la cuestión económica, otras modalidades y prácticas limitan una aparente «democratización» de los estudios en el extranjero, las cartas de recomendación, necesarias para postular a una universidad o conseguir una beca, reproducen distinciones entre estudiantes de universidades más o menos prestigiosas. La universidad de estudios de pregrado es, de hecho, otro indicador de orígenes sociales y si bien las universidades privadas también tienen becas que permiten diversificar el origen social de sus estudiantes, los costos siguen siendo en muchos casos elevados para muchas familias, sobre todo tratándose de universidades ubicadas en la parte noroeste de la proyección.

Gráfico 4: ACM. Proyección de las variables ilustrativas (socio-generacionales). Ejes 1 y 2.

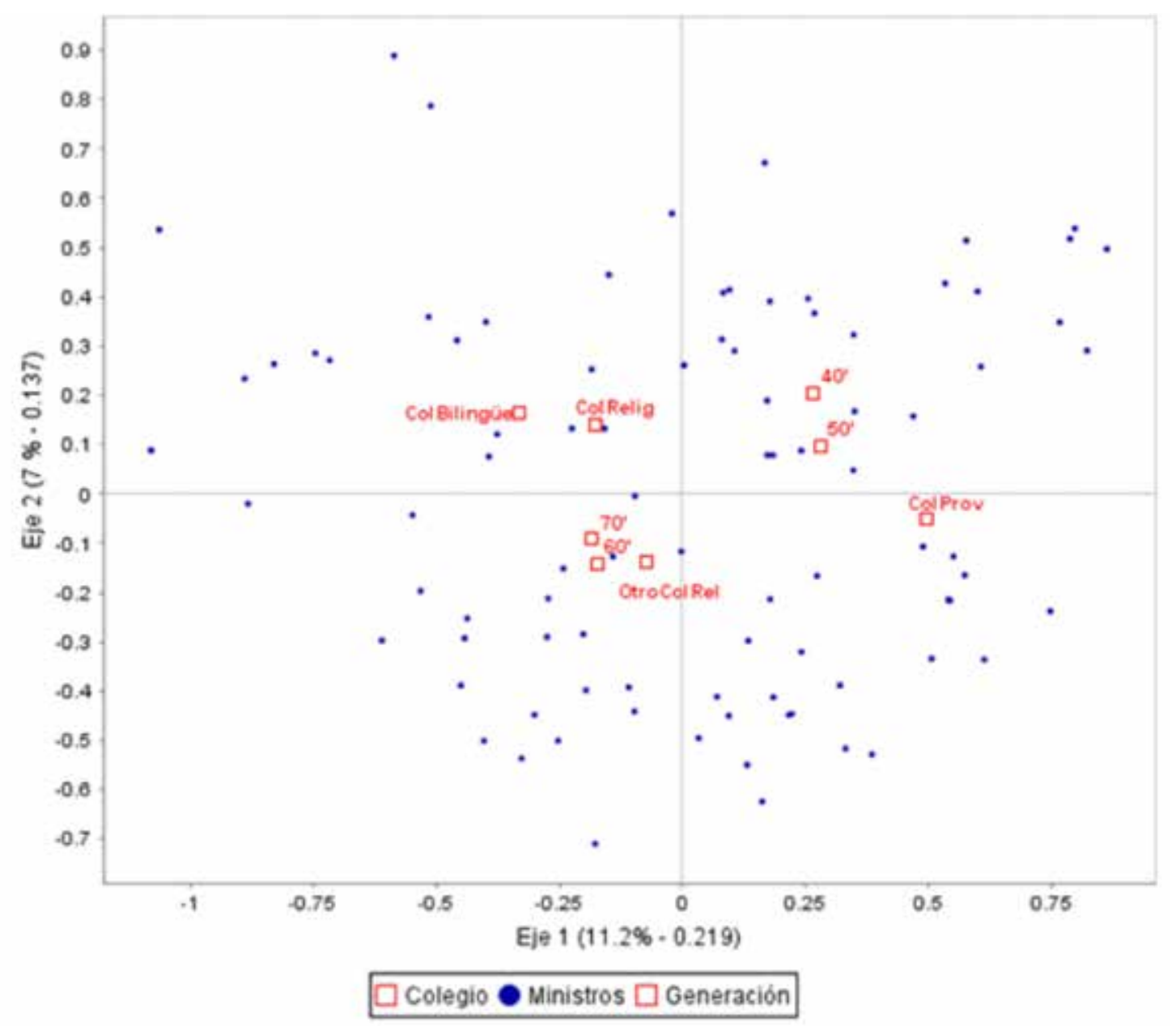


Por otro lado, la dimensión generacional es visible en la estructuración del espacio. Al proyectar una variable ilustrativa y representar la década cuando nacieron los ministros, se puede notar una diferencia entre quienes nacieron en los años $1940-1950$ y las generaciones de 1960 y 1970. La figura del ministro-ingeniero se relaciona a personas nacidas en décadas anteriores a la de los ministrosgerentes. Esta diferencia es clara si recordamos que el $43 \%$ de los ministros con un perfil de «gerente» (trayectoria ascendente en el sector privado hasta llegar a posiciones gerenciales) nacieron entre los años 1960-1970 (76\% si incluimos los años 50), mientras que el 53\% de los ministros-empresarios (cuya posición en el sector privado se debe en primer lugar a inversiones personales o familiares) son de la generación de 1930-1940. La estructura generacional aquí presentada reproduce dinámicas que se dieron en los espacios de la competencia económica. La figura del gerente y del consultor ganaron peso en el sector privado en la década de los 80, lo cual tiene que ver con cambios que se dieron en la estructura de la competencia económica; tales como la financiación de las actividades de los principales grupos económicos, las alianzas estratégicas crecientes con grupos extranjeros y la incorporación de prácticas y saberes provenientes de los EstadosUnidos (Cotler, 1998; Durand, 2017). Esta diferenciación generacional en el perfil de los ministros peruanos refuerza nuestra hipótesis de una relación, al menos parcial, de homología entre dinámicas que se dan en la formación de las élites económicas y las altas esferas político-administrativas del país. Más allá de una presentación de casos individuales, el ACM permite asociar estas nuevas figuras a cambios que se dieron en las estrategias de reproducción social de las élites económicas, como el peso creciente de un capital social y cultural fuertemente internacionalizado, reemplazando las estrategias de transmisión familiar del capital económico (recordando dinámicas que se dieron en otros países, como Bolivia o Mexico: Poupeau, 2004; Chardavoine, 2020).

Mediante estas figuras, podemos contextualizar el cambio de la posición de los ministros peruanos en relación con los partidos políticos. El primer eje está, como lo habíamos mencionado, construido en parte por esta oposición entre perfiles políticos y no políticos, lo cual se inscribe dentro de dinámicas generacionales. Así, las posiciones partidarias se encuentran en gran parte asociadas a este perfil del ministro ingeniero, empresario o independiente del sector industrial o agricultura, mientras que la ausencia de vínculo político es más frecuente dentro de los ministros jóvenes, cuya trayectoria en el sector privado se hizo en grupos financieros o de consultoría económica, ocupando importantes puestos de dirección o gerencia en dichas empresas. La caída del peso político y simbólico de los partidos políticos en el Perú desde finales de los 80, tiene como consecuencia, en este sentido, el alejamiento de las generaciones más jóvenes de la militancia política partidaria, a diferencia de la socialización política que pudieron tener élites ministeriales en otros países. En este aspecto, la «tecnocratización» de las élites ministeriales peruanas no tiene 
solamente que ver con un alejamiento de dichas élites de la política, sino más bien con estas nuevas estrategias que hemos mencionado, de consagraciones políticoadministrativas en un contexto de deslegitimación importante de los partidos políticos y de cambios ocurridos en las esferas económicas.

\section{La composición socio-profesional de los ministerios}

Una vez identificadas las principales líneas de fuerza alrededor de las cuales se construyen las figuras ministeriales en el Perú, es posible ver cómo los ministerios estudiados se ubican en dichos ejes. En otras palabras, se trata de ver si algunos ministerios suelen caracterizarse por un conjunto de particularidades específicas, proyectando en el espacio una serie de variables ilustrativas que representan la participación en los ministerios estudiados.

Gráfico 5: ACM. Proyección de las variables ilustrativas (ministerios). Ejes 1 y 2.

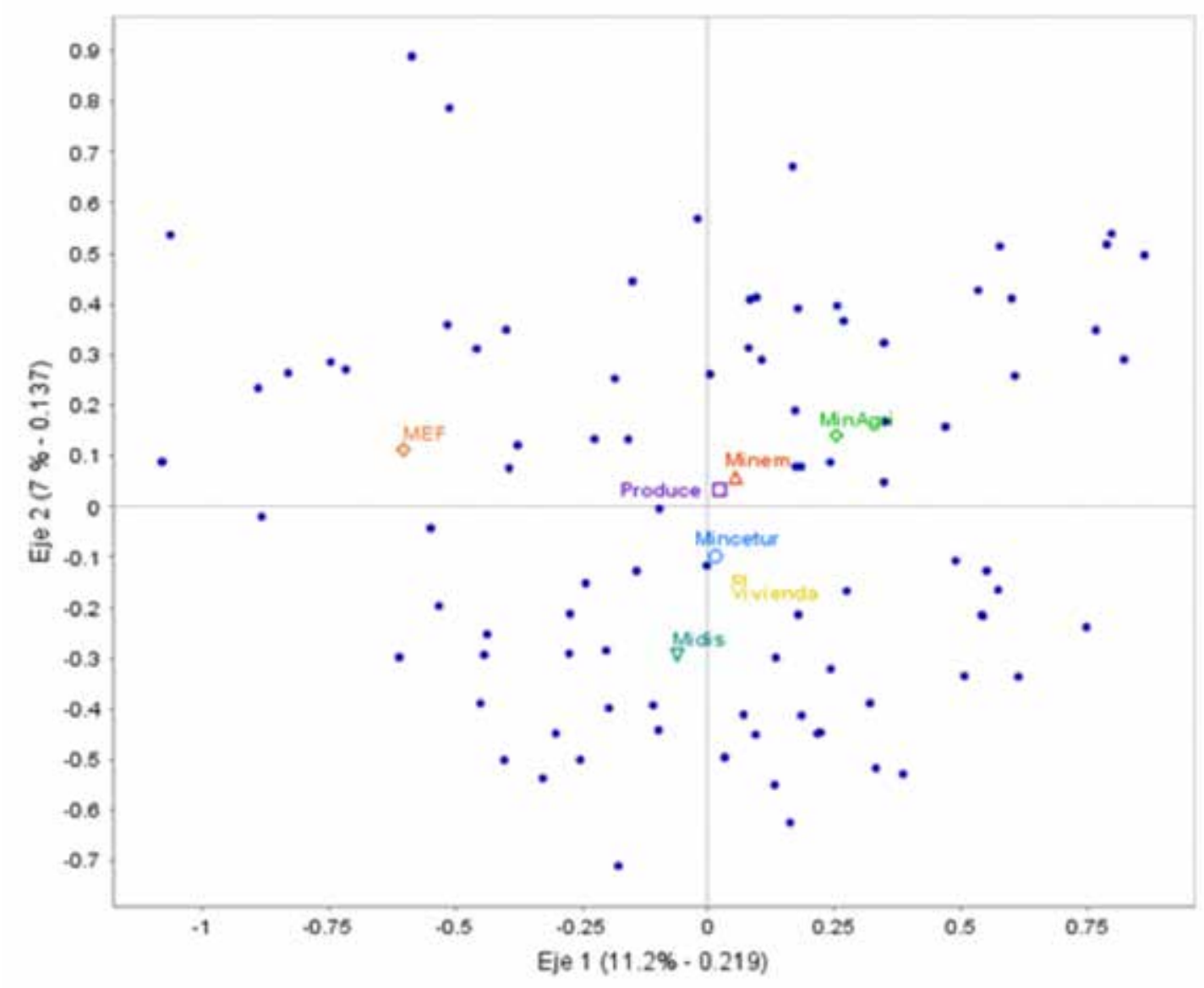

Ministros $\square$ Produce $\bigcirc$ Mincetur $\triangle$ Minem $\diamond$ Minagri $\square$ Vivienda $\&$ MEF $\nabla$ Midis

El elemento central de diferenciación entre los ministerios se expresa netamente en el segundo eje (vertical), recordando que el perfil del alto-funcionario en cada uno de los ministerios se debe en gran parte a su posición socio-profesional anterior y al conjunto de sus capitales adquiridos mediante estas trayectorias. Por 
lo tanto, ministerios como el MEF, Minagri, Minem o Produce se caracterizan por cercanías más importantes con el sector privado que el Mincetur, Vivienda y el Midis. En cuanto al primer eje, este aísla al MEF por un lado y, en menor medida, al Minagri. Se puede, una vez identificada la posición general de cada institución en la estructura de los ministerios, encontrar algunas dinámicas de cambio dentro de algunos ministerios, los cuales tienen que ver con las dinámicas socio-generacionales ya mencionadas, pero también con los cambios que se dan en el campo político (cambios de presidencias).

La posición del Ministerio de Agricultura se destaca así por su especificidad, con una importancia pronunciada de ingenieros agrónomos con trayectorias empresariales y vínculos políticos, especialmente en la primera década de los años 2000. Álvaro Quijandría, dos veces ministro de agricultura durante la presidencia de Toledo, hermano de otro ministro (Jaime Quijandría, en el MEF y el Minem), ilustra bastante bien esta figura del ingeniero-empresario con vínculos políticos: miembro del directorio y gerente de empresas agrícolas, ha sido vicepresidente de la Confederación Nacional de Instituciones Empresariales Privadas (Confiep), pero también presidente de la Asociación Nacional de Empresarios Agrarios del Perú. Proviene de una familia ligada a los negocios agrícolas, estudió en el colegio Maristas en Lima y se vincula tempranamente a la política. En 1983 es candidato a regidor en Cañete para el Partido Popular Cristiano y luego candidato a congresista para el Frente democrático (1990), Renovación (1995) y Perú Posible (2000 y 2001). Otros ministros como Rafael Quevedo, Luis Ginnochio, Juan José Salazar o Miguel Caillaux tienen características relativamente parecidas (empresarios o ejecutivos en el sector agrícola, muchas veces con vínculos políticos), aunque también encontramos empresarios de otros rubros (como Ismael Benavides o Eduardo Gonzales). A partir de la presidencia de Ollanta Humala, con el nombramiento de Milton Von Hesse, se puede observar cierta evolución en el perfil general de los ministros de Agricultura. Es así que, entre especialistas de proyectos de inversión (Von Hesse o José Arista) y gerentes multi-situados como Manuel Benites Ramos o José Manuel Hernández Calderón, el cambio antes mencionado en la estructura de las posiciones ministeriales es bastante visible en este período.

El Ministerio de Producción es un ministerio «joven», que reúne desde julio de 2002 las competencias del antiguo ministerio de Pesca, así como funciones relativas a cuestiones industriales anteriormente asociadas al Ministerio de Comercio exterior. ${ }^{7}$ Encontramos desde la creación del ministerio con Eduardo Iriarte, Javier

7. Dicho sea de paso, la creación de este ministerio es reveladora de los cambios que ocurrieron en el Perú. A inicios de 1980, las competencias relativas al comercio exterior y a la «integración» estaban en disputa entre el Ministerio de Economía y el Ministerio de Industrias. Hoy en día, el Ministerio de Comercio Exterior tiene una institucionalización sólida mientras que el Ministerio de Producción se encarga de las cuestiones industriales, entre otras. 
Reátegui, David Lemor o Alfonso Velásquez, hasta estos últimos años con Pedro Olaechea o Bruno Giuffra, un importante número de ministros-empresarios con vínculos políticos y gremiales. ${ }^{8} \mathrm{Si}$ bien esta es una característica predominante del Ministerio de Producción, los diferentes ciclos políticos, así como los cambios en la estructura de la competencia económica en el país, introdujeron una diversificación en el perfil de dichos ministros, desde gerentes con conexiones políticas (Rafael Rey y Daniel Córdova) hasta profesionales y ejecutivos de alto nivel en grupos extranjeros (Lienecke Schol o Piero Ghezzi).

El Ministerio de Economía y Finanzas es otro ministerio cuya posición en el espacio es fuertemente marcada, recordando que las principales características de los ministros de Economía en los años 2000 no se limitan a altas credenciales académicas ni tampoco a posiciones en organismos internacionales, sino también, y de manera central, a carreras realizadas en el sector financiero, grupos extranjeros y grandes consultorías económicas. Así, la gran mayoría de los ministros que ocuparon la cartera de Economía y Finanzas en el transcurso de los años 2000 tienen una carrera que se caracteriza por una circulación entre estos «universos». Respecto al perfil general de estos ministros y especialmente a su carrera privada, se debe recalcar la importancia relativamente nueva de economistas provenientes de las áreas de estudios económicos o estudios de mercado y riesgo de la banca comercial privada (Tuesta y Carranza en el BBVA, Cooper y Segura en el BCP), lo cual introduce un cierto cambio con la figura «tradicional» del banquero de inversión (Kuczynski, y de alguna manera Silva Ruete o Thorne), pero también del empresario integrado en importantes actividades y gremios nacionales (Ismael Benavides). Sin entrar en detalles, podemos resaltar una vez más el interés de incluir las diferentes carreras y trayectorias privadas de los ministros en el análisis, porque son imprescindibles para entender las características, los recursos y los saberes incorporados en la institucionalización de estas figuras ministeriales. Esta perspectiva permite, por ejemplo, evidenciar la fuerte diferenciación que podemos encontrar en el perfil de los ministros de Economía y los ministros de Desarrollo e Inclusión Social, a pesar de que estos ministerios son presentados a menudo como arquetípicos de la «tecnocracia» peruana.

El Ministerio de Desarrollo e Inclusión social aparece claramente como el menos ligado a posiciones en el sector privado, o al menos en importantes puestos ejecutivos o de gerencia. El Midis ha sido frecuentemente presentado como un ejemplo de "tecnocratización» de la cuestión social, impulsado por una generación de economistas del desarrollo social (Vela et al., 2014), provenientes en gran parte de la Universidad Católica del Perú, pero también influenciado en su estructuración por el Ministerio de Economía y Finanzas (Vergara \& Encinas,

8. En una gran mayoría gremios empresariales del sector industrial (SNI) o de exportaciones y comercio (ADEX y Cámaras de Comercio). 
2016). A diferencia de los demás ministerios estudiados, el Midis es, por mucho, el ministerio con más presencia femenina, de los siete ministros presentes en la base de datos, seis son mujeres. El nombramiento a este puesto de dos ministras involucradas en cuestiones de salud (Liliana La Rosa y Ariela Luna) representa un cambio en la estructuración del ministerio, con la participación de personas con un perfil caracterizado por la intervención social «directa» (ambas trabajaron para asociaciones del sector salud) más que por estudios de pobreza, desde enfoques generalmente económicos. El hecho de que estas dos ministras fuesen nombradas en la presidencia Martín Vizcarra y que, de igual manera, las dos figuras con importantes trayectorias en el sector privado (Cayetana Aljovín y Jorge Meléndez) hayan sido nombradas por Pedro Pablo Kuczynski, ilustra de manera clara que las características generales de los ministros en el Perú también están sujetas a cambios, según orientaciones del ejecutivo, lo cual resalta el carácter siempre político de los nombramientos ministeriales.

Para objetivar dichos cambios y dinámicas, hemos proyectado, aquí también de manera ilustrativa, modalidades que representan la presidencia bajo las cuales los ministros estudiados estuvieron en sus respectivos puestos.

Gráfico 6: ACM. Proyección de las variables ilustrativas (presidencias). Ejes 1 y 2.

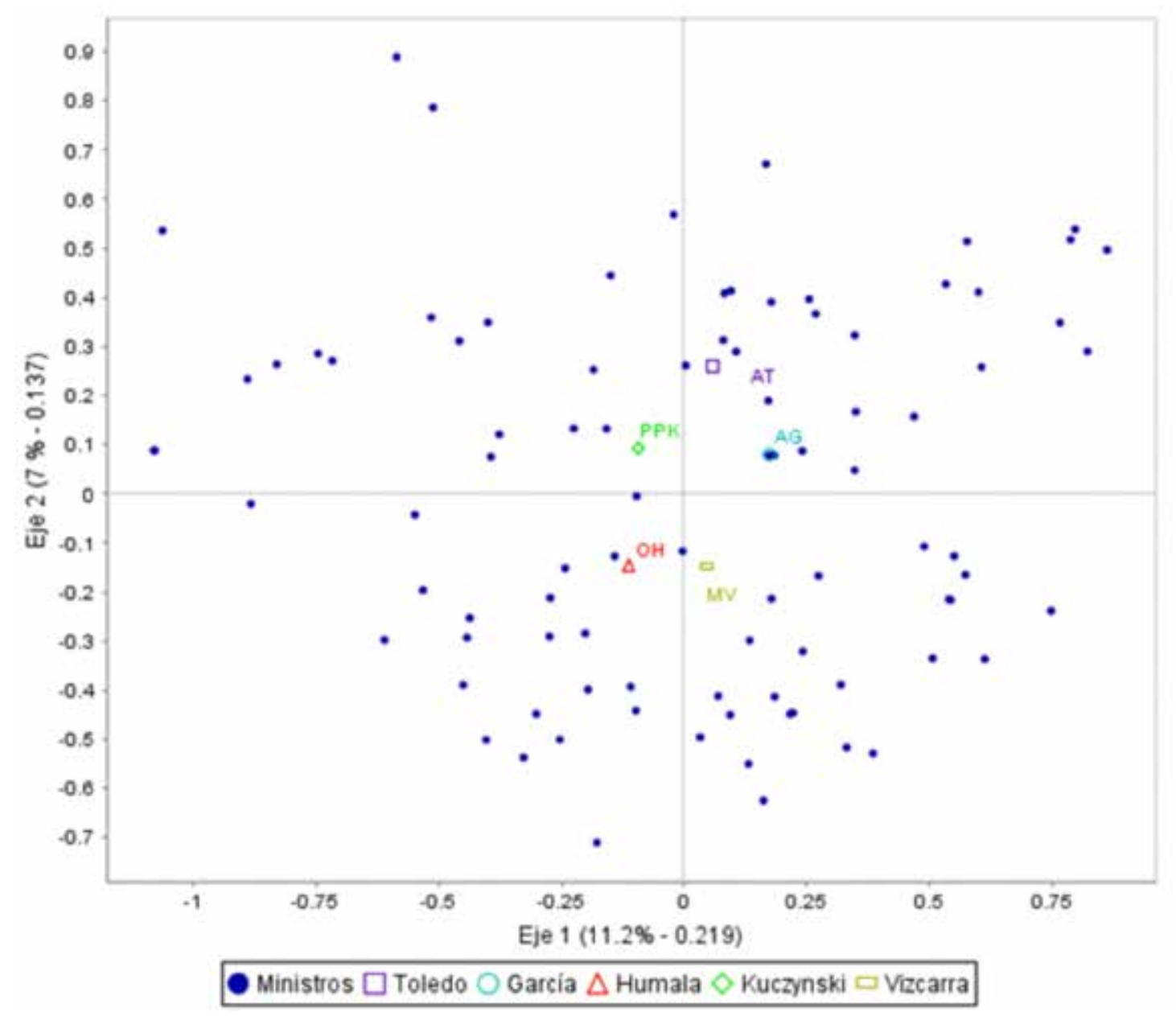


La proyección de las variables ilustrativas permite ver una diferencia neta en el perfil general de los ministros en función a las presidencias a las cuales pertenecieron. Vemos así muy claramente que Alejandro Toledo, Alan García y Pedro Pablo Kuczynski recurrieron a ministros más ligados a importantes actividades privadas que Ollanta Humala o Martín Vizcarra. Si bien las características del «gabinete de lujo» de Pedro Pablo Kuczynski son bastante similares al perfil socio-profesional del mismo Kuczynski como ministro, la posición de las otras presidencias dentro del espacio ilustra igualmente su grado de cercanía e integración con sectores importantes del campo del poder en el Perú. Ollanta Humala, pero también en alguna medida Martín Vizcarra representan cada uno a su manera cierta lejanía con sectores «tradicionales» de la vida político-económica del país: Humala por su imagen de político «independiente» y Vizcarra por su perfil de político regional poco integrado a esferas limeñas. Por el contrario, tanto García como Toledo o Kuczynski representaron, en su momento, una opción política más cercana a algunos sectores tradicionales de la vida política peruana, lo cual no quiere decir que encarnaban en ese entonces la figura del establishment, pero sí que se beneficiaron de amplias alianzas.

Por lo tanto, este criterio de diferenciación entre presidencias va más allá de una oposición entre izquierda y derecha (probablemente poco significativa en los gobiernos peruanos durante las dos últimas décadas). Refleja, en gran parte, la posición misma de los diferentes presidentes peruanos en el campo político y el campo del poder peruano. Alejandro Toledo es bastante interesante al respeto por ser un político relativamente nuevo en la escena política para la elección del 2001 y establecer alianzas tanto con sectores de centroizquierda como con figuras reconocidas de las derechas como Raúl Diez Canseco Terry (su primer vicepresidente) o Pedro Pablo Kuczynski (jefe del plan de gobierno). Esta relativa cercanía con algunos de estos sectores importantes en el campo del poder peruano se objetiva aquí por el perfil general de los ministros, y permite matizar y profundizar el conocimiento que tenemos de los cambios en los espacios de producción de las políticas públicas en el país. El perfil «técnico» de la presidencia de Humala no se debe solamente a los cambios en los procesos de constitución de una «carrera pública informal» (cambios identificados y presentados, entre otros, por: Grompone Velásquez, 2017 retomando conceptos de Dargent, 2015) sino también son el resultado, siempre provisional, de las pugnas entre estos grupos socio-profesionales, en un período caracterizado por una presidencia relativamente «atípica», al menos tratándose de su posición en los espacios de poder en el país.

De esta manera, la elección de Pedro Pablo Kuczynski en el 2016 se tradujo en el corto período de su presidencia efectiva, por un cambio no solamente en el perfil general de los ministros, sino también en algunas propuestas de gobierno: bajo el lema de «destrabar» la inversión pública y privada, se introdujeron cambios en 
las reglas de inversión pública en el Plan Nacional de Diversificación Productiva, pero también en las reglas de las Alianzas Público-Privado. Los conflictos abiertos entre los ministros salientes de Humala y los ministros de los primeros gabinetes de la presidencia Kuczynski, permiten evidenciar una oposición que va más allá de una diferencia entre «técnicos» y políticos. Abarca una diferenciación en el perfil profesional de estos ministros, que solo hemos podido presentar a grandes rasgos y que se podría profundizar mucho más.

\section{Conclusión y perspectivas para el estudio del poder en el Perú}

El estudio de los ministros y de las posiciones ministeriales en el Perú es un campo de investigación importante para entender quiénes construyen el Estado en el Perú, preguntándose cómo se forjan y cambian las principales figuras del poder político-administrativo. Lejos de ser una posición puramente técnica, una cartera ministerial es una posición de poder político. En este sentido, objetivar las características de quienes acceden a estos puestos permite mejorar el conocimiento que tenemos no solamente de los ministros sino también de la estructura de posiciones de quienes construyen el Estado (o pretenden hacerlo), su relación con otros espacios y esferas del campo del poder en el país, evitando así abstracciones («el Estado», «los políticos», «los empresarios»; «los tecnócratas», etc.).

Hemos resaltado aquí la importancia no solamente de las características técnicas y educativas de los ministros, sino también de la importancia de trayectorias en importantes sectores privados. La objetivación de una aparente relación de homología entre dinámicas generacionales que se dieron en el campo económico y esta estructura de posiciones ministeriales recuerda que no se puede pensar y estudiar a las élites estatales en el Perú como grupos totalmente independientes. Esto no quiere decir que no existen dinámicas, tendencias y tentativas para institucionalizar una nueva forma de "hacer» las políticas públicas en el país, y tampoco que estaríamos aquí frente a un Estado totalmente capturado por grupos económicos. Sin embargo, el análisis permite recordar que importantes puestos ministeriales están fuertemente ligados a posiciones, saberes y prácticas provenientes del sector privado. Estos saberes y prácticas no son únicos ni tampoco uniformes, cambian, son objetos de oposiciones y visiones divergentes y tiene diferente importancia en cada ministerio.

El estudio de los ministros es una forma de aproximarse a esos saberes y pugnas entre grupos socio-profesionales Son la cara más visible de las élites estatales y, por lo tanto, su designación está fuertemente asociada a estrategias de legitimación. Sin embargo, el conocimiento de quiénes hacen al Estado no se puede limitar a estas figuras. Observar otros niveles de gobierno, así como algunas «zonas grises» del Estado, permite tener una visión más amplia, identificando por ejemplo las relaciones de homología que existen entre estos niveles y espacios. 


\section{Referencias bibliográficas}

Alvarez Rodrich, A. (1993). El poder en el Perú (Apoyo SA). Lima: Editoriales Apoyo.

Ballatore, M., \& Blöss, T. (2008). L’autre réalité du programme Erasmus: Affinité sélective entre établissements et reproduction sociale des étudiants. Formation emploi. Revue française de sciences sociales, (103), 57-74. Recuperado de http:// journals.openedition.org/formationemploi/2478

Behr, V., \& Michon, S. (2013). The representativeness of French cabinet members in the Fifth Republic: A smokescreen? French Politics, 11(4), 332-355. Recuperado de https://halshs.archives-ouvertes.fr/halshs-01009729

Benites Alvarado, A., \& Hurtado Paz, F. (2018). Mirando más allá de lo técnico: La importancia del factor político en el desarrollo de las políticas públicas en el Perú 2013-2016. Politai: Revista de Ciencia Política, 9(17), 40-89.

Bourdieu, P. (2017). La distinción: Criterio y bases sociales del gusto. Barcelona: Taurus.

Bourdieu, P., \& Saint Martin, M. de. (1976). Anatomie du gout. Actes de la Recherche en Sciences Sociales, 2(5), 2-81. https://doi.org/10.3406/arss.1976.3471

Bustamante, B. G. (2013). Factores de acceso y permanencia de la élite política gubernamental en Chile (1990-2010). Política. Revista de Ciencia Política, 51(1), 119-153. https://doi.org/10.5354/rp.v51i1.27436

Chardavoine, J. (2020). Las élites corporativas de México (2000-2015): Dinámicas del capitalismo familiar. Revista Española de Sociología, 29(3).

Conaghan, C. M., Malloy, J. M., \& Abugattas, L. (1990). Business and the «Boys»: The Politics of Neoliberalism in the Central Andes. Latin Americain Research Review, 25(2), 3-30.

Cotler, J. (1998). Los empresarios y las reformas económicas en el Perú. Lima: IEP, Instituto de Estudios Peruanos.

Crabtree, J., \& Durand, F. (2017). Perú: Élites del poder y captura política. Red para el Desarrollo de las Ciencias Sociales en el Perú.

Dargent, E. (2012a). El Estado en el Perú: Una agenda de investigación. Pontificia Universidad Católica del Perú. Escuela de Gobierno y Políticas Públicas. Recuperado de http://repositorio.pucp.edu.pe/index/handle/123456789/136843

Dargent, E. (2012b). Technocracy under democracy: Assessing the political autonomy of experts in latin america (PhD Dissertation). The University of Texas at Austin, USA. 
Dargent, E. (2014). Technocracy and democracy in Latin America: The experts running government. Cambridge: Cambridge University Press.

Dezalay, Y., \& Garth, B. G. (2002). La internacionalización de las luchas por el poder: La competencia entre abogados y economistas por transformar los Estados latinoamericanos (C. A. Rodríguez Garavito, Ed.). Bogotá: Inst. Latinoamericano de Servicios Legales Alternativos [u.a.].

Dubois, V. (2015). La acción del Estado, producto y objeto de disputa de las relaciones entre espacios sociales. Sudamérica : Revista de Ciencias Sociales, 1(4), 18-33. Recuperado de https://fh.mdp.edu.ar/revistas/index.php/sudamerica/ article/view/1556

Dulong, D. (1996). Quand léconomie devient politique. La conversion de la compétence économique en compétence politique sous la Ve République. Politix. Revue des sciences sociales du politique, 9(35), 109-130.

Durand, F. (2008). Las nuevas élites del poder: Sueños económicos y pesadillas políticas. En L. Pásara (Ed.), Perú en el siglo XXI (1. ed, pp. 71-97). Lima: Fondo Editorial, Pontificia Universidad Católica del Perú.

Durand, F. (2017). Los doce apóstoles de la economía peruana: Una mirada social a los grupos de poder limeños y provincianos (Primera edición digital). Lima, Perú: Fondo Editorial, Pontificia Universidad Católica del Perú.

Fourcade-Gourinchas, M., \& Babb, S. L. (2002). The Rebirth of the Liberal Creed: Paths to Neoliberalism in Four Countries. American Journal of Sociology, 108(3), 533-579.

Gayo, M., Teitelboim, B., \& Méndez, M. L. (2013). Exclusividad y fragmentación: Los perfiles culturales de la clase media en Chile. Universum (Talca), 28(1), 97-128. Recuperado de https://www.academia.edu/3985775/Exclusividad y fragmentaci\%C3\%B3n los perfiles culturales de la clase media en Chile

Grompone, R. (Ed.). (2016). Incertidumbres y distancias: El controvertido protagonismo del Estado en el Perú. Lima: IEP, Instituto de Estudios Peruanos.

Grompone Velásquez, Á. (2017). La inacabable búsqueda de eficiencia: Claves para entender las agendas tecnocráticas en el Perú actual. En Á. Grompone Velásquez \& A. Gálvez (Eds.), Burócratas y tecnócratas: La infructuosa búsqueda de la eficiencia empresarial en el Estado peruano del Siglo XXI (pp. 99-213). Lima: IEP, Instituto de Estudios Peruanos.

Heredia, M. (2015). Cuando los economistas alcanzaron el poder: O cómo se gestó la confianza en los expertos. Buenos Aires, Argentina: Siglo Veintiuno Editores. 
Ihl, O. (2012). Objetividad de Estado. Sur la science de gouvernement des Chicago Boys dans le Chili de Pinochet. Revue internationale de politique comparée, 19(3), 67-88.

Le Roux, B. (2014). Analyse géométrique des données multidimensionnelles. Paris: Dunod.

Le Roux, B., \& Rouanet, H. (2009). Multiple Correspondence Analysis (1. a ed.). SAGE Publications.

Oré, M. T., \& Rap, E. (2009). Políticas neoliberales de agua en el perú. Antecedentes y entretelones de la ley de recursos hídricos. Debates en Sociología, 0(34), 33-66.

Poupeau, F. (2004). Sur deux formes de capital international. Les «élites de la globalisation» en Bolivie. Actes de la recherche en sciences sociales, 151-152(1), 126.

Poupeau, F., O’Neill, B. C., Cortinas Muñoz, J., Coeurdray, M., \& BenitesGambirazio, E. (2020). The field of water policy: Power and scarcity in the American southwest (1 Edition). London; New York: Routledge, Taylor \& Francis Group.

Rentería, M., Grompone Velásquez, A., \& Reátegui Amat y León, L. (2020). Educados en el privilegio: Trayectorias educativas y reproducción social de las élites en Perú. Revista Española de Sociología, 29(3).

Román, A., \& Ramirez Villarán, M. A. (2018). ¿El mito sigue vivo?: Privatización $y$ diferenciación social en la educación peruana. Lima: IEP, Instituto de Estudios Peruanos.

Sala i Vila, N. (2016). Saberes, ciencias y política en la formación del Estado en el Perú. En R. Grompone (Ed.), Incertidumbres y distancias: El controvertido protagonismo del Estado en el Perú (pp. 147-171). Lima: IEP, Instituto de Estudios Peruanos.

Savage, M., Devine, F., Cunningham, N., Taylor, M., Li, Y., Hjellbrekke, J., ... Miles, A. (2013). A New Model of Social Class? Findings from the BBC's Great British Class Survey Experiment. Sociology, 47(2), 219-250. Recuperado de http:// journals.sagepub.com/doi/10.1177/0038038513481128

Tanaka, M. (Ed.). (2010). El estado, viejo desconocido: Visiones del estado en el Perú (1. ed). Lima: IEP, Instituto de Estudios Peruanos.

Tanaka, M., Vera Rojas, S., \& Barrenechea, R. (2009). Think tanks y partidos políticos en el Perú: Precariedad institucional y redes informales. En E. Mendizabal \& K. Sample (Eds.), Dime a quién escuchas... Think tanks y partidos políticos en América Latina. Lima, Perú: IDEA Internacional \& ODI. 
Vela, E., Becerra, M. G., García, S., \& Roca, P. (2014). Tecnocracias sociales: El surgimiento de una tecnocracia en el Ministerio de Desarrollo e Inclusión Social. Social Technocracies: The emergence of a technocracy in the Ministry of Development and Social Inclusion. Politai: Revista de Ciencia Política, (9), 83-104.

Vergara, A. (2012). Alternancia sin alternativa. ¿Un año de Humala o veinte años de un sistema? Revista Argumentos, año 6(3), 1-13.

Vergara, A., \& Encinas, D. (2016). Continuity by Surprise: Explaining Institutional Stability in Contemporary Peru. Latin American Research Review, 51, 159-180.

Villagarcia, P. A. S. (2014). ¿Todos los 'hombres' del Presidente?: Una mirada al Poder Ejecutivo a propósito de los ministros y ministras en el Perú post-Fujimori (2001-2014). Politai: Revista de Ciencia Política, (9), 29-57.

Vivanco, C. (2002, julio 15). Un gabinete con «rostro» político. Semana Económica.

Wise, C. (2003). Reinventando el Estado: Estrategia económica y cambio institucional en el Perú (1. ed). Lima: Universidad del Pacifico, Centro de Investigación.

Zárate, P., \& Rentería, M. (2019). Una mirada a la dimensión simbólica de las clases: Revista Argumentos, año 13(1), 63-71.

Zavaleta, M. (2014). Coaliciones de independientes: Las reglas no escritas de la politica electoral (Ed.1). Lima: IEP Instituto de Estudios Peruanos. 